\title{
Enabling Responsible Online Gambling by Real-time Persuasive Technologies
}

\author{
George Drosatos $^{1 \star}$, Fotis Nalbadis ${ }^{1}$, Emily Arden-Close ${ }^{1}$, Victoria Baines ${ }^{1}$, Elvira Bolat ${ }^{1}$, \\ Laura Vuillier ${ }^{1}$, Theodoros Kostoulas ${ }^{1}$, Marcin Budka ${ }^{1}$, Sonia Wasowska ${ }^{2}$, Maris Bonello ${ }^{3}$, \\ Jamie Brown ${ }^{4}$, Tessa Corner ${ }^{5}$, John McAlaney ${ }^{1}$, Keith Phalp ${ }^{1}$, and Raian Ali ${ }^{1}$ \\ ${ }^{1}$ Bournemouth University, Talbot Campus, Fern Barrow, Poole BH12 5BB, UK \\ ${ }^{2}$ Sky Betting \& Gaming, 2 Wellington Place, Leeds, West Yorkshire LS1 4AP, UK \\ ${ }^{3}$ Kindred Group, Fawwara Buildings, Msida Road, Gzira GZR 1405, Malta \\ ${ }^{4}$ William Hill, Greenside House, 50 Station Road, Wood Green, London N22 7TP, UK \\ ${ }^{5}$ StreetScene Addiction Recovery, Allington House, 46 DeanPark Road, Bournemouth BH1 1QA, UK \\ (gdrosatos, fnalmpantis, eardenclose, vbaines, ebolat, lrenshawvuillier, tkostoulas, mbudka, \\ jmcalaney, rali, kphalp) @bournemouth.ac.uk, sonia.wasowska@skybettingandgaming.com, \\ Maris.Bonello@kindredgroup.com, jbrown@williamhill.co.uk, tessa@streetscene.org.uk
}

\begin{abstract}
Online gambling, unlike other offline addiction forms, provides unprecedented opportunities for monitoring users' behaviour in real-time, along with the ability to adapt persuasive interactions and messages that would match the gamblers usage and personal context. Online gambling industry usually offers Application Programming Interfaces (APIs) that are mainly intended to allow third-party applications to interact with their services and enhance user's experience. In this article, we claim that such API's can also be utilised to retrieve gamblers' online data, such as browsing and betting history and other available offers, and use it to build more proactive and intelligent responsible gambling systems. We report on our experience in this field and make the argument that the available data for persuasive marketing and usability should, under certain usage conditions, also be made available for responsible online gambling services. We discuss the psychological foundations of our proposed approach and the risks and challenges typically identified when building such a software-assisted intervention, persuasion and emotion regulation technology. We also explain the potential impact of corporate social responsibility and data protection prospects. Furthermore, we explore the required principles that should be followed by the gambling industry for enabling responsible online gambling. We finally propose a conceptual architecture to show our vision and explain how it can be implemented. In the broader context, the article is intended to provide insights on building behavioural awareness and regulation information systems related to problematic digital media usage.
\end{abstract}

Keywords: Persuasive Technologies, Responsible Online Gambling, Gambling Data Availability, Corporate Social Responsibility.

\footnotetext{
* Corresponding author

(C) 2018 George Drosatos et al. This is an open access article licensed under the Creative Commons Attribution License (http://creativecommons.org/licenses/by/4.0).

Reference: G. Drosatos, F. Nalbadis, E. Arden-Close, V. Baines, E. Bolat, L. Vuillier, M. Budka, S. Wasowska, M. Bonello, J. Brown, T. Corner, J. McAlaney, K. Phalp, and R. Ali, "Enabling Responsible Online Gambling by Real-time Persuasive Technologies," Complex Systems Informatics and Modeling Quarterly, CSIMQ, no. 17, pp. 44-68, 2018. Available:
} https://doi.org/10.7250/csimq.2018-17.03

Additional information. Authors ORCID iD: G. Drosatos - https://orcid.org/0000-0002-8130-5775, R. Ali https://orcid.org/0000-0002-5285-7829. PII S225599221800099X. Received: 15 October 2018. Accepted: 26 November 2018. Available online: 31 December 2018. 


\section{Introduction}

Online gambling is on a continuous escalation because it is easily accessible and is enhanced with innovative information technologies that are able to predict behaviours, personalise the medium and make it progressively appealing and fascinating to users. Also, gambling disorder is now recognised by the DSM- $5^{1}[1]$ as a behavioural disorder. The total annual gross gambling yield (Apr 2016 Mar 2017) of the Great Britain gambling industry was 13.8 billion British pounds ( $2 \%$ increase from Apr 2015 - Mar 2016), respectively, in worldwide was 284 billion British pounds. At the same period, the active accounts of the online/phone gambling consumers were 28.87 million, showing an increase of $25.3 \%$ from the previous period, and there are more than 400,000 UK citizens with self-identified problem gambling [2]. Thus, there is an unprecedented opportunity to try to tackle problem gambling and also make the gambling experience more informed and conscious, through the retrieval of online gambling behaviour data and its intelligent and proactive real-time usage.

The scale and complexity of the problem becomes even higher through the ubiquitous accessibility of such betting services over the internet compared to the traditional gambling which requires going to betting shops. Such techniques, along with peer pressure, may elongate exposure, stimulate relapse and block attempts to adjust gambling to an acceptable level. In addition, the utilisation of persuasive techniques (e.g. leader boards and badges) in online gambling may increase the risk of gambling to be used as compensation for perceived failure in the real world, mitigate low self-esteem and are a substitute for lack of social skills.

The features that make online gambling more attractive and exciting, at the same time also have a great potential to fight the gambling problem. The accessibility and persuasive techniques utilised in online gambling can evenly be used when building persuasive techniques to moderate against the problematic behaviour. Actually, cyberspace provides a unique opportunity to enhance classical behaviour change as it offers interactivity, real-time response, traceability of use data and support for personalisation and context-awareness. Based on the established research in influence [3], help-seeking and behaviour change [4], online addiction labels [5] and persuasive elements of online peer groups [6], we advocate persuasive techniques for assisting responsible online gambling behaviour rather than relying solely on coercive ones. Also, this argument shall recognise the risk factors when applying such technology for addictive behaviours [7].

Self-regulation technologies are advocated to fight problematic online behaviour given the nature of the medium and the availability of different solutions when classic and coercive approaches are enacted. Self-regulation techniques are focused on users who have an active role in changing their own behaviour. A basic assumption and precondition would be that people would recognise the benefits of achieving the behavioural change and would, consequently, actively seek help. Moreover, such self-regulation approaches when used to regulate online problematic behaviour may benefit from the medium itself, in order to monitor behaviour and introduce mitigation technologies when problems occur or are about to happen, e.g. interactive warning labels and persuasive interventions such as timers and avatars [5]. An intervention could be designed based on the Theory of Planned Behaviour [8], which holds this attitude towards a behaviour, subjective norm (perception of how others feel about the same behaviour) and perceived control over a behaviour influence on the intention to perform that behaviour, which eventually influences whether the individual performs the behaviour. Feedback about normality of gambling and amounts gambled relevant to others could help individuals to control their behaviour, in accordance with theory around social norms. Correspondingly, awareness of how behaviour varies across contexts (e.g. making people aware of how their gambling differs based on time and place) could grow perceived control over gambling.

${ }^{1}$ The Diagnostic and Statistical Manual of Mental Disorders, Fifth Edition (DSM-5) is a taxonomic and diagnostic tool published by the American Psychiatric Association (APA) that defines and classifies mental disorders in order to improve diagnoses, treatment and research. 
Otherwise, there are several arguments about the strength and risks associated with self-regulation through technology. We still do not have high scientific evidence of their effectiveness and also, particularly, of the sustainability of the change they may bring [9]. For instance, delivering interventions within peer group settings could potentially be harmful due to factors related to the structure and dynamics of a group. This may sometimes lead to strengthening the negative behaviour [10] and encouraging social loafing [11] or facilitate a temporary change that is encouraged by the need or pressure to comply [12]. Persuasive technologies can cause individuals to feel frustrated, anxious, pressured by peers and guilty when they do not comply with the system or have to deceive it [13]. Despite the high potential of using online gambling behaviour data in order to build persuasive techniques that will help gamblers to regulate their gambling, caution is required, as there is a risk that the change may go in unpredictable directions or be costly in terms of triggering other behaviours. It is therefore necessary to proceed with caution.

In this article, we argue the case for enabling responsible online gambling with persuasive technologies that utilise the gamblers' online behavioural, emotional and profile data. For instance, the real-time collection of a piece of information such as "the gambler has reached the monthly spending limit, is logged in, is navigating through the deposit page" can be used to trigger a message visualising their past betting behaviour and a reminder of a commitment already made. Also, other more complex scenarios can be envisaged. We are aware that many gamblers may not recognize that they are addicted to gambling, and therefore less likely to engage with real-time information. However, (1) they may nonetheless be aware that they are demonstrating problematic behaviour, and (2) we aim to reach individuals whose gambling is still under control. Making such data available will significantly enhance the positive image of online gambling sites as being managed by caring and responsible operators [14]. Online gambling behaviour data is a rich source for tailoring offers and marketing material in order to fit the potential interests of a gambler. There could be a fine line between the legitimate marketing and content customisation on the one hand and mis-selling and manipulation on the other. To increase fairness and transparency, we suggest that this same data is also made available in the same timing to support gamblers, their counsellors and their surrogate software, for the purpose of responsible and informed gambling and also a transparent gambling experience. This does not exclude further data that may be only of use for responsible and informed gambling and be offered solely for that purpose. Our argument is concentrated on the practical experience we have in the context of the EROGamb project ${ }^{2}$ supported by three main gambling operators in Europe and two problem gambling recovery centres in the UK. The project is funded by a major independent charity for commissioning gambling research in the UK.

The article is organised as follows. We discuss psychological grounds in behaviour change and their manifestations when applied to online and real-time style in Section 2. We give a particular emphasis to the role of behavioural data which can infer the emotions and mental state of the gambler and how this can be used for persuasive techniques in Section 3. We discuss concerns in relation to commercially sensitive data and corporate social responsibilities in Section 4. Then, we discuss the rights of users to retrieve their behavioural data and enable their persuasive responsible gambling software in Section 5. We explore the required principles that should be followed by the gambling industry for enabling responsible online gambling in Section 6 . We propose a representative conceptual architecture of such persuasive information systems in Section 7. Finally, we discuss the current stand of the gambling industry in this area and the challenges and risks associated with it in Section 8, and present thoughts and future research directions in Section 9.

\footnotetext{
${ }^{2}$ EROGamb Project: https://research.bournemouth.ac.uk/project/erogamb/
} 


\section{Real-time Persuasive Intervention: Psychological Foundations}

Psychological research has determined a number of factors that explain individual's behaviour. This research is the basis of theories such as the aforementioned Theory of Planned Behaviour and others such as Protection Motivation Theory [15]. These theories share a number of characteristics. This includes factors such as whether the behaviour is viewed positively or negatively (i.e. attitude); our perceived social norms of the attitudes and behaviours of those around us; and our perceived ability to engage in the behavioural change, considered in terms of perceived self-efficacy or behavioural control. A key element of these theories is the concept of perception. Perception - our understanding of how others around us think and behave, along with our understanding of ourselves, may not be accurate. This is supported by research into cognitive biases, such as the fundamental attribution error [16], in which we mis-attribute the reasons for others' actions. Perceptions can be malleable, and therefore there is potential to target these in order to create behaviour change. However, understanding the principles of behaviour does not always allow for the simple application of behaviour change strategies, due to factors such as the gap between intention and behaviour [17]. Models such as the transtheoretical model [18] aim to explain how to prompt behaviour change, yet there remain practical barriers to the implementation of these models. Digital technologies and their information systems allow for new methods of behaviour change and prevention strategies. For instance, digital monitoring of behaviour and automated real-time intervention has successfully encouraged drivers to stay within speed limits [19]. This is an example of the principles of implementation intention [20], which states that in general people aim to behave in a positive way, but fail to do so due to factors such as competing demands on their attention or forgetfulness in executing the behaviour within the correct content. This quantification of behaviour enhances several established behaviour change strategies.

Another important underlying factor in behaviour and behaviour change is motivation. This refers to the reason for individual's actions and needs, often considered in terms of intrinsic and extrinsic motivation. Intrinsic motivation relates to the desire to engage in action for the personal enjoyment or satisfaction of having done so, without any external pressures. Extrinsic motivation on the other hand refers to engaging an action because of an external factor, e.g. provision of a reward such as a payment. The role of motivation in behaviour can be counter-intuitive. For instance, it has been noted that individuals may be more likely to complete a task if they are not expecting a financial payment for doing so [21], possibly because the presence of an external motivator reduces the intrinsic motivation an individual may feel for completing that task. Development of successful interventions facilitated by techniques such as gamification may require preliminary processes. First education may be required to strengthen intrinsic motivation [22] for the behaviour in question (desire to carry out a behaviour based on internal rewards). This can be done by requiring individuals to select their individual reasons (from a list) for performing a particular behaviour. Given the interactive nature of the online medium, users could request to be reminded of those reasons on screen at key points while engaging in a particular behaviour.

Within behaviour change interventions, goal setting theory is used to encourage individuals to strive to achieve a certain outcome, which may itself involve a series of behavioural changes. This technique is most successful when the behavioural changes are measurable or observable [23]. This is particularly relevant to behaviours that can be tracked and shared using digital technologies. Goal setting can be guided by rules such as the SMART (specific, measurable, agreed upon, realistic and time-bound) criteria [24]. This holds that goals are most likely to be met when they are specific (target a specific area for improvement), measurable (quantify indicators of progress), achievable (can realistically be met by the setter), relevant (apply to the situation in question) and time-bound (are required to be completed within a certain amount of time) [25]. Combined with this, advice on developing implementation intentions (in situation Y, I will do goal-directed behaviour X), significant by enhance goal attainment and is effective in promoting goal striving, preventing goal pursuit from unwanted influences and reducing disengagement [26]. 
An important part of goal setting is to provide feedback to the individuals on how well they are progressing towards their goal [25]. If it is correctly done (e.g. individuals are given feedback that is tailored to their progress, constructive, and focuses on behaviours and strategies) this can increase the individuals self-regulation or ability to control their own behaviour and sense of self-efficacy [25] (how much they believe that they are capable of changing their own behaviour), further motivating them to achieve behaviour change. In offline settings however, the opportunities for the individual to receive feedback on their process may be limited; for instance, someone aiming to reduce gambling activity may only receive feedback during weekly meetings with a counsellor or support group. In contrast, feedback on progress that is being measured continuously can be delivered immediately. Indeed, the feedback may even be delivered whilst the behaviour in question is occurring, if the technology is able to detect this. For instance, gamblers could receive pop-up messages alerting them to the fact that they have exceeded previously set time limits. This ability to intervene in real-time with the behaviour is novel in the field of behaviour change, where interventions and feedback are typically asynchronous, occurring after the behaviour has occurred.

Goal setting techniques can be further enhanced through appropriate use of social comparisons as a persuasive tool, which digital technologies are again particularly suited to support. Social comparison can be a powerful influence on behaviour, with individuals driven to determine their place within a social system, and to reach their desired position within this system [27]. Leader boards can be used to present individuals with information about how their status and progress compare to fellow group members. Digital technologies can be used to not only provide individuals with continual and easy access to the leader boards, but also to tailor the group membership to that which is the most appropriate for the individual. Individuals tend to set a goal of being near the top of a leader board if they are presented with one [28]. Signifiers of success and social status can also be used to enhance behaviour change, such as the use of digital badges to indicate that an individual has achieved a particular goal [28]. This relates to social norms, which refers to what people perceive as typical behaviours, attitudes and aspirations of those within their peer groups. However, these norms can be significantly misperceived and overestimated within groups, particularly those involved in risky or addictive behaviours [29]. Challenging and correcting these misperceptions through social comparisons has been used to bring behaviour change techniques to problematic gambling [30].

Technology can increase the potential of behaviour change techniques, but also the potential for unintended consequences. We suggest some potential ways to prevent this below with the study of emotions involved in gambling and persuasive information systems.

\section{Emotions Regulation \& Persuasive Responsible Gambling}

In Section 2, we discussed how persuasive information systems can be involved in changing the behaviour of gamblers. However, emotions are intrinsically linked to behaviours and one cannot fully understand gambling behaviours without taking into account the emotion that may be driving the behavior. For instance, gambling is thought to increase arousal and decrease negative emotions [31], [32]. The team authoring this article include gambling therapists, responsible gambling officers and researchers with direct relation to problem gamblers and observe that negative emotions, such as depression, guilt, anxiety and negative avoidance, and also positive emotions, such as excitement, are both an integral part of the gambling experience. Moreover, recent research shows that emotion disregulation may be involved in the development and the maintenance of gambling disorder [33], [34], [35]. Despite their fundamental role, research on emotions in the gambling literature is scarce. Particularly, it is currently unclear: (a) what type of emotions are experienced during different phases of gambling, (b) which are the ones particularly associated with particular aspects such as loss chasing or near wins and near losses, and (c) what is the best way to capture them. The latter point is of particular importance as individuals with 
a gambling addiction tend to report high level of alexithymia, manifested by difficulties with identifying and describing one's emotions [33], [36].

In this context, developing a multimodal interface which can help identifying disordered patterns of gamblers is important. In particular, a combination of different multimodal measures, such as facial expressions and galvanic skin response, can help us better understand the role of emotions in gambling, as well as the different manifestations of the gambling experience. To do so, the following signals can be analysed: electrodermal activity [37], [38]; facial electromyography [39]; electroencephalography [40]; acceleration [41], [42]; speech [43], [44] and face [45], [46]. While some of the sensors might be obtrusive, their usage during the testing phase will allow identifying the different responses a person might express [47]. This information is useful, even if that specific sensor is not used in the initial prototype. For instance, it is true that the use of electromyography sensors is obtrusive. However, when placed on the face, they would allow identifying the facial features which are important. The later information can be used for fine-tuning the models utilized for facial emotion recognition. Moreover, despite many sensors are currently obtrusive, the progress of sensor data acquisition technologies is nowadays enabling non-obtrusive acquisition of more physiological and behavioural data. A straightforward example can be the physiological and behavioural sensors existing in everyday devices (such as accelerometer, gyroscope, heart rate and electrodermal activity sensors embedded in a mobile phone or in a smart-watch).

From an affective computing point of view, attempting to capture gambling emotions results in several open issues that need to be investigated, such as the synchronous capture of physiological and behavioural signals and the identification of the most important modalities for emotion detection while gambling [41], [42], [43], [48]. Moreover, we need to identify how the different modalities correlate during an experience, e.g. the correlation between galvanic skin response and facial expressions (intrapersonal correlations) [49]. The proposed solution would be focused on answering the following questions:

- Which are the emotions experienced by gamblers?

- Which are the appropriate signal cues for capturing these emotions?

- How can we automatically detect emotions in gambling, using multimodal data?

- How can we use data mining methods to identify the links between the physiological responses and the gambling emotions?

In order to analyse the different multimodal signals and identify an emotion representation, signal acquisition, pre-processing and feature extraction of the physiological and behavioural signals will take place. Factor analysis and/or principal component analysis will be utilised for identifying the appropriate representation of the gambling emotions and identification of the modalities that represent most of the variance of the emotional data. In this way, it will be possible to investigate the complex links between multimodal responses and emotions. The challenge in the development of the emotion models relies on accounting for the different reaction time for different modalities, the different duration of different emotions and periods of loss of control, as well as the available data for building the corresponding models. To respond to those challenges the necessary machine learning techniques will be explored, such as support vector machines [50] for handling high dimensional data, hidden Markov models [51] for creating temporal models, as well as deep learning approaches [52].

Besides their role in gambling, emotions also have their own place in persuasive information systems. For instance, sending emotionally stimulating messages (such as reminding the gambler of familial impact or longer term financial issues) may help facilitate responsible gambling [53], particularly if they are delivered at the right moment, in synchrony with their online gambling behaviour. Similarly, recent research has shown that sending personal feedback to online gamblers seems to reduce time and money spent on gambling [54]. Further exploration could involve looking at whether sending this feedback at a particular time during the gambling process (such as during loss chasing or denial) may influence the emotions involved and ultimately further 
increase responsible gambling. Moreover, it is important to also assess the emotions that may be involved with such persuasive messages, to make sure this technology facilitates - and not impairs - behaviour change. These questions need yet to be investigated. The rise in smartphone technology and its potential use in psychiatry and health behaviour [55] means that this is the perfect time to do so. Indeed, one could hope that smartphone technology will help with the automatic identification of physiological, emotional and behavioural signals, so that persuasive techniques can be implemented at the right time, when the individuals are in the right emotional state, and with the right level of motivation. This could help prevent some of the unintended consequences of persuasive technology.

\section{Corporate Responsibility vs. Commercially Sensitive Data}

Technological solutions are the heart of online gambling services and products [14]. Such solutions are based around data capture and use that enables personalized and immersive gambling experiences [56]. Responsible gambling, which assumes "safe gambling product or environment" [57], is mostly used by gambling operators to evidence their involvement with corporate social responsibility (CSR) practices. Online gambling operators are deemed to act responsibly to manage societal expectations and contentious perceptions of the gambling industry and in particular tackle issues of problem gambling [58]. To date, common CSR practices exercised by gambling operators are principles of transparency usually associated with informed choice available to gamblers and prevention of harm linked to control over gambling features and content [59]. However, literature [60] suggests that scientific evidence, demonstrating consensus about the effectiveness of currently in use responsible gambling measures to prevent the incidence or reduce the prevalence of gambling-related harms, is limited.

It is evident that most CSR practices are linked to technological essence of online gambling products and services. In this process, data management and sharing are pivotal to CSR and duty of care conduct. Despite existing literature [14], [56] arguing that data can aid responsible gambling decision making, surprisingly, the allowance of gambling operators of the retrieval of online gambling behaviour data by responsible gambling software is hardly part of any currently practiced responsible gambling activities. This could be explained by the commercial sensitivity of these data especially when the software is operated by third parties. Moreover, as commercial entities gambling operators may be wary of engaging with a responsible data sharing process because of an industry rivalry where customer data and customers behavioural data is considered as a source of competitive advantage. Literature argues [61] that any data and information that has economic value or potential to cause economic harm to an organisation can be classified as commercially sensitive data. The underlying logic of commercially sensitive data is that some data requires protection and 'secret' status to forest innovative business practices [62]. Examples of such data in online gambling industry are marketing strategies, consumer lists and online behavioural data. However, despite being viewed commercially as sensitive customer data, in light of EU General Data Protection Regulation [63] they are considered as personal data, ownership of which lies with the gambling customers themselves. Upon customer's individual request customer behavioural data can and have to be shared with anyone. Moreover, the counter argument is that since the financial terms of many online gambling products are known within the industry, the argument that data availability and transparency would cause competitive harm seems weak. In fact, a disclosure of anonymised consumer lists or behavioural data may be required to serve a greater societal interest [62].

Corporate social responsibility in a digital era is an area of continuous change due to ongoing emergence of new digital technology applications such as the use of artificial intelligence in customer services and in the profiling of consumers. Legal developments might be slower to regulate and even understand application of technology, whereas organisations need to lead the change vis-à-vis development of so-called ethical practices and principles in moral use of data. Conversations around ethical big data are contributing towards a shared understanding 
of ethical, social, and legal complexities of data analytics [64]. Moreover, within the online gambling discipline there is a movement towards establishing CSR principles and encouraging data sharing and transparency. For instance, academic researchers [65], [66] are exploring ethical recommender systems to provide guidance on the moral norms for serving recommendations of online gambling products to players. Although such ethical recommender systems research is in its infancy stage, it triggers industry-wide conversations about online gamblers' moral obligations to prevent mis-selling, behavioural manipulations and privacy intrusion [67].

In this article we, therefore, argue that it is data usage, not data sharing, that generates a competitive value. Data sharing on the long run presents an advantage to the in industry, hence, a balanced approach to data sharing is necessary. This is particularly true for the online gambling context where, while making profits, gambling operators are expected to protect gamblers, and detect vulnerable and problematic behaviour [57]. In fact, responsible gambling strategies resulting from credible use and sharing of data can form an individual operators' competitive differentiation as well as improve the industry's image and drive sustainable business growth (see Figure 1).

Moreover, we are proposing a collaborative approach to data sharing and management, at least within a single operator's context if not across the industry. In particular, we argue the case for a principle that data made available for marketing divisions and their software would need to be equally, subject to usage conditions, available to responsible gambling divisions and their surrogate software. Responsible gambling divisions can then enact certain configurations for each sector of the gamblers. The principle is to provide equal opportunity for both directions and leave the decision to the gamblers themselves. Following this principle, gambling operators are managing all three elements crucial to an excellent customer experience, compliance process and CSR: entertainment, accessibility, and risk.

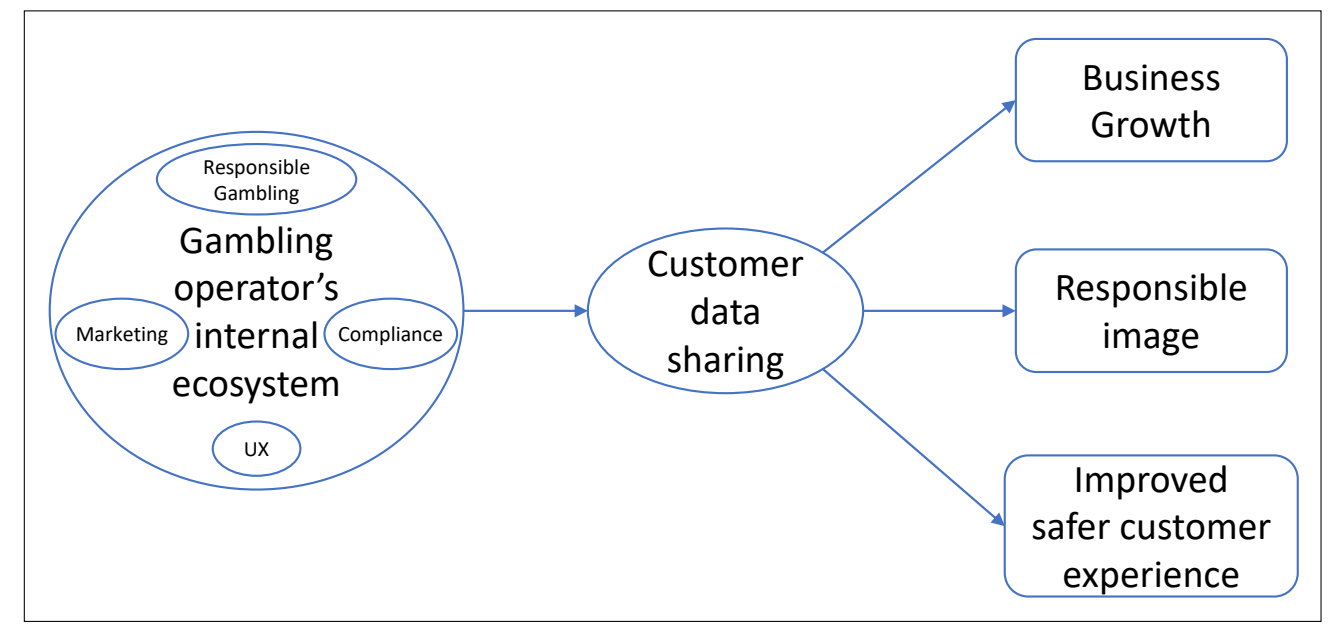

Figure 1. Data sharing as principle of responsible gambling provision

\section{Online Gambling Behaviour and Data Protection Legislation}

Much has been made in recent public discourse of the requirements on data processors of the European Union General Data Protection Regulation (GDPR). This legislative instrument was adopted on 27th April 2016 and implemented on 25th May 2018, and applies to data processors and data controllers who provide services to EU citizens, regardless of the geographical location of that entity's chief operations [63]. This section asserts that, far from constraining the ability of gambling operators to share behavioural data with responsible gambling persuasive software, landmark data protection legislation not only permits this sharing, but also empowers users to invest and engage in the process to the potential benefit of their own wellbeing. 
EU Member States have been required to transpose the provisions of GDPR into national law, but have enjoyed some flexibility in how they do so. This has led to differences in those national provisions over which Member States have been able to exercise greater discretion, e.g. the age at which young people can access online services without parental consent (Article 8). While multinational gambling operators inevitably need to give due consideration to the potential for differences between specific national provisions, the principles of 'lawfulness, fairness and transparency' (Article 5.1(a)) underpin all the major innovations presented in GDPR. These include the requirement for a Privacy Impact Assessment to be conducted on any proposed new collection and processing of data.

Procedures must also be in place to allow data subjects to request copies of their personal data processed by a particular service ("Subject Access Requests"). GDPR calls out in particular the requirement to show greater transparency on "the existence of automated decision-making, including profiling", and to provide "meaningful information about the logic involved, as well as the significance and the envisaged consequences of such processing for the data subject" (Article 15.1(h)).

In addition, Article 22.1 gives the data subject the right to object to being profiled: "The data subject shall have the right not to be subject to a decision based solely on automated processing, including profiling, which produces legal effects concerning him or her or similarly significantly affects him or her".

GDPR does not prohibit online gambling operators from sharing data with responsible gambling software with the intention of benefiting a user's wellbeing, provided:

- A Privacy Impact Assessment has been conducted on the collection and processing of the data.

- Information concerning "the purposes of the processing for which the personal data are intended as well as the legal basis for the processing" and "the recipients or categories of recipients of the personal data, if any" is adequately communicated to the subject (Article 13.1(c),(e)).

- The user has freely given unambiguous and informed consent to the described data processing and transfer.

This last point is one of the key themes of GDPR. It permeates the text of the regulation, but is perhaps set out most in section 32 of the Preamble: "Consent should be given by a clear affirmative act establishing a freely given, specific, informed and unambiguous indication of the data subject's agreement to the processing of personal data relating to him or her, such as by a written statement, including by electronic means, or an oral statement. This could include ticking a box when visiting an internet website, choosing technical settings for information society services or another statement or conduct which clearly indicates in this context the data subject's acceptance of the proposed processing of his or her personal data. Silence, pre-ticked boxes or inactivity should not therefore constitute consent."

In regard to the processing of online gambling behavioural data for marketing and for responsible gambling purposes, GDPR requires a granular approach to the giving of consent: "Consent should cover all processing activities carried out for the same purpose or purposes. When the processing has multiple purposes, consent should be given for all of them. If the data subject's consent is to be given following a request by electronic means, the request must be clear, concise and not unnecessarily disruptive to the use of the service for which it is provided."

The importance of informed consent and transparent communication has been underlined in the recent public discourse surrounding reported large scale data breaches. A progress report by the UK Information Commissioner's Office on its ongoing investigation into the alleged misuse of Facebook user data has highlighted the legal requirement for consent that is informed by transparent communication on data processing: "People cannot have control over their own data if they don't know or understand how it is being used. That's why greater and genuine transparency about the use of data analytics is vital" [68]. 
Informed and unambiguous consent to profiling by responsible gambling software, in fact, promises to be more than merely a matter of legal compliance. The requirement to give explicit consent empowers users to volunteer for analysis, which in turn may lead to greater levels of self-awareness and behavioural change. In regard to industry regulation, the UK Gambling Act 2005 points to the Data Protection Act 1998 for matters of data processing and transfer (c. 29) [69], and the UK National Responsible Gambling Strategy highlights the need for harm minimisation interventions [70]. In this respect it may be argued that consent to profiling for the purpose of improving public health is within the spirit of the law and government efforts at both national and international level.

\section{Principles for Enabling Responsible Online Gambling}

We advocate a principle of fair and equal opportunity data sharing. The data that is associated with the gambler and their behaviour and are used for marketing and personalising content should be made available to the gamblers and their authorised representatives, such as family members and counsellors, in an accessible and useful manner and through automated means to enable scalability and rapid processing. In practical terms, this means the data should be subject to retrieval by automated and programming means (such as Application Programming Interfaces (APIs)) and would also need to be put in place for the use by third-party applications or other beneficiaries (e.g., family members, counsellors and therapists) when authorised by the gambler. In Figure 2, we show our vision about the data flow from the gambling industry (and other possible resources) to the gamblers and/or other third-party beneficiaries.

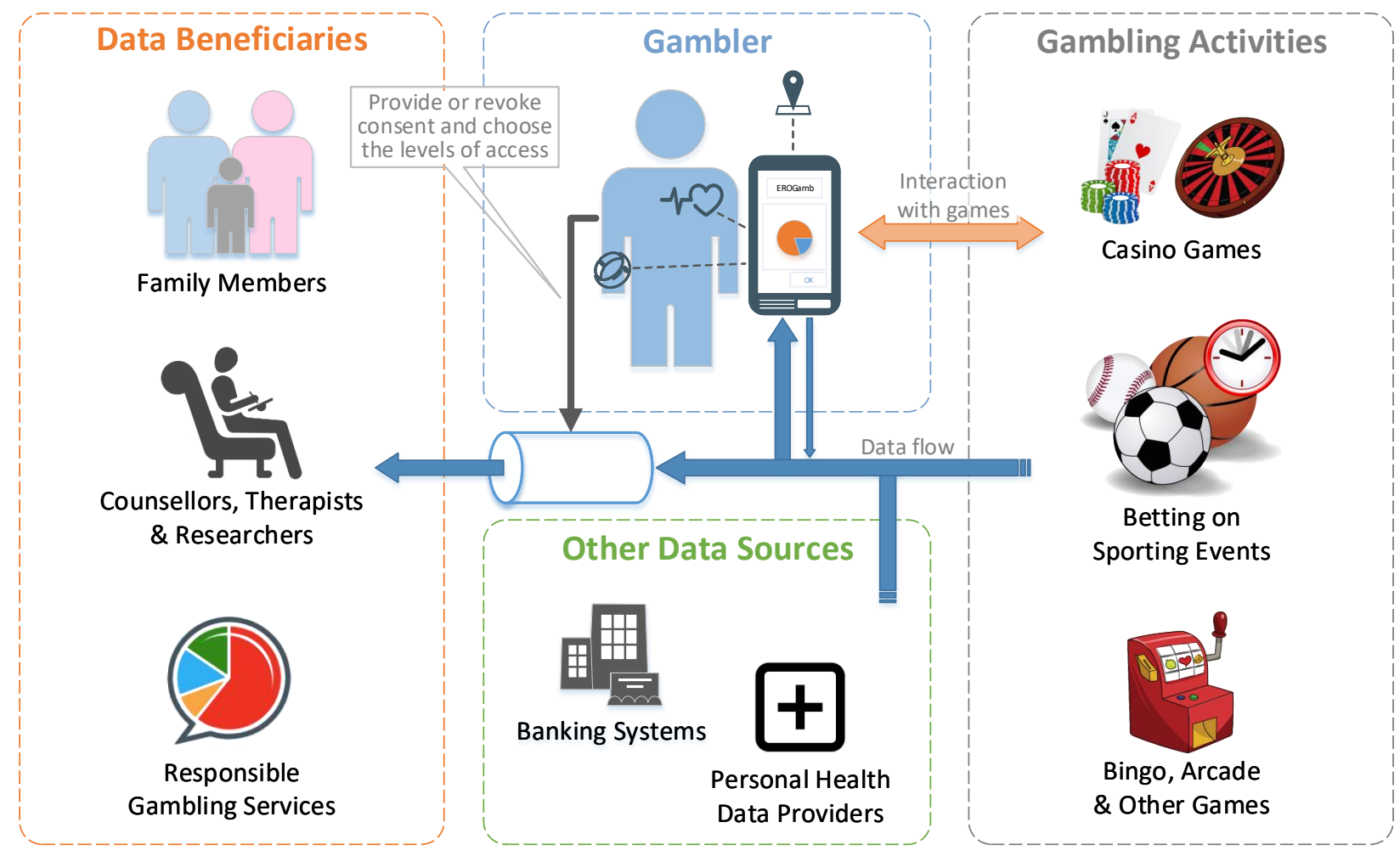

Figure 2. The data flow of gamblers to third-parties

Common obstacles relate mainly to potential privacy issues (see details in Section 5) even when informed consent is given, the coverage by existing legislative frameworks for data protection and the potential competitive disadvantages that such data provisioning can introduce to operators. Given the advantages and also the increased demand for transparency, this would eventually become a recommended practice for demonstrating advanced corporate social responsibility and 
enhancing the image of the gambling industry by inspiring the trust of the public and clients through responsible, caring operations (see details in Section 4).

We call on the future online gambling websites and applications to provide such data to gamblers and their authorised surrogate software, helpers and counsellors in an automated and real-time (or close to real-time) style, in line with the requirements of data protection regulations. This greater transparency from operators regarding the gambling experience will enable greater awareness of gambling behaviour in gamblers. In order to explore the required principles that should be followed by the gambling industry and their responsible gambling offices, we carried out a cooperative study including interdisciplinary experts. The followed methodology of this study is presented in Subsection 6.1.

\subsection{Methodology}

The required principles are derived following a cooperative work amongst the authors as part of the EROGamb project ${ }^{3}$. The authors' team includes interdisciplinary expertise including software engineering, psychology, social informatics, security and privacy, corporate social responsibility, behavioural economics, marketing and responsible gambling practises. The principles are refined iteratively to cater for the multiple viewpoints, exceptions and the removal of potential ambiguity. A national workshop was then held on 25th April 2018 to discuss the project and the semi-final version of these principles. Before the actual workshop, they were made available on a shared online document for commenting for two weeks. The workshop and the online commenting process included representatives from three gambling operators, three UK-based research groups working on gambling and cyberpsychology and three major charities working on gambling recovery and responsible gambling in the UK. Demographic information for the workshop's participants is presented in Table 1, where we only count the participants' main expertise and all participants are familiar with the gambling domain and the idea of EROGamb project.

Table 1. Demographic information of the workshop's participants

\begin{tabular}{|l|l|l|l|}
\hline Variable & Value & $\boldsymbol{n}=\mathbf{2 5}$ & $\mathbf{\%}$ \\
\hline Sex & Male & 15 & $60 \%$ \\
& Female & 10 & $40 \%$ \\
\hline Years of experience & $<5$ & 5 & $20 \%$ \\
& $5-10$ & 11 & $44 \%$ \\
& $>10$ & 9 & $36 \%$ \\
\hline Academic experience & Yes & 20 & $80 \%$ \\
& No & 5 & $20 \%$ \\
\hline Field of expertise & Computer \& Data Science & 4 & $16 \%$ \\
& Software Engineering \& Informatics & 3 & $12 \%$ \\
& Security \& Privacy & 5 & $20 \%$ \\
& Psychology & 9 & $36 \%$ \\
& Regulatory Compliance & 4 & $16 \%$ \\
\hline
\end{tabular}

\subsection{The Principles}

The derived principles of this process are focused for the gambling industry and their responsible gambling offices in relation to empowering consciousness and gambling awareness with meaningful and easy access to gambling behavioural data. These principles are 12 and are presented below.

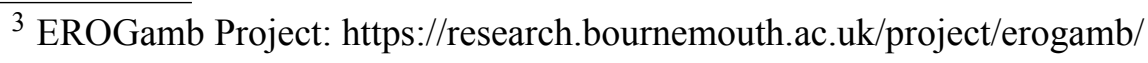




\section{Principle 1: Recognised Benefits}

Responsible gambling and gambling behavioural awareness benefit from online gambling behaviour data as well as other relevant non-play data (for common types of data, see Section 7.2).

\section{Principle 2: Multi-Beneficiary and Multi-Stakeholder Processes}

Data beneficiaries are diverse, e.g. gamblers, family members, counsellors and responsible gambling divisions. The data, after processing, is meant to help involved parties in assuring responsible and informed gambling. The gambler's explicit consent is required on an individual basis for each other party to obtain access to the data.

\section{Principle 3: Accessible Data Provisioning and Integrated Design}

Data should be accessible via programming means (such as Application Programming Interfaces (APIs) or equivalent), thereby allowing it to be processed in a timely and scalable manner for the benefit of gamblers and their authorised parties. These programming means could be standardised, so that the data is accessed in the same way regardless of the operator and that they all make the same information available (or there might be different tiers/levels of data sharing, too). The design of third-party applications should be reviewed to ensure that neither user experience nor the relevant data protection rules are compromised (such as GDPR-compliant in EU [63]).

\section{Principle 4: Authentication and Informed Consent}

Gambling operators should authenticate gamblers and their authorised parties. Gamblers should be authorised (according to the comprehensive legislation) to provide or revoke consent to responsible and informed gambling software that receives the data, irrespective of whether this software belongs to the operator or a third-party (see Section 7.1 for an exemplary authentication and authorisation process).

\section{Principle 5: Transparency of Service Existence and it Purpose}

To inspire consumer confidence in the industry, and to enable broader outreach for responsible and informed gambling, gambling operators should actively make gamblers aware of the existence of the service, i.e. the possibility to collect their online gambling behaviour data to enable a more responsible and informed gambling experience.

\section{Principle 6: Complementary Data Sources}

To better understand gambling behaviour, complementary data sources should be envisaged, subject to informed consent being given for each specific use. These may be provided by gamblers or the more extensive infrastructure. For instance, mobile devices may indicate mood, smart watches may indicate heart rate, and banking systems may indicate financial status (see Section 7.2 for examples of such data). The data collection should be done under the gamblers' agreement, and the usage of it should be explicitly defined and compliant with the data protection regulations.

\section{Principle 7: Granular Data Access}

Gamblers should be able to choose the levels of access granularity when sharing online gambling behaviour data. This includes a time frame, data types, the frequency of sharing, and recipient parties and allowed usage. Gambling operators and other authorised data users are expected to make the consequences of access decisions visible to gamblers to help them make informed decisions, e.g. the limitations incurred by withholding some data.

\section{Principle 8: Acknowledged Approximation}

Because of the margin for error when interpreting or making judgements about behaviour from complementary sources, gambling operators, gamblers and third-parties accept that no final judgement can be made solely based on such data. As a corollary, all parties acknowledge that recommendations are not an exact science, and feedback should be obtained from gamblers to revise them. 


\section{Principle 9: Acknowledging Potential Unintended Consequences}

Irrespective of data processing neutrality, goodwill and informed consent provided, unintended consequences can occur. These may occur due to technical problems and psychological side effects of information communicated to gamblers, e.g. lowering self-esteem due to visualisation of gambling data indicating a repetitive failure to meet reduction goals. These unintended consequences need to be accounted for through basing the solutions on established research and best practice.

\section{Principle 10: Complementary Approaches}

Obtaining and using online gambling behaviour data complements other approaches for responsible gambling, such as motivational interviewing and cognitive behaviour therapy. Gamblers should be aware that collecting online gambling behaviour data is not a substitute for other approaches.

\section{Principle 11: Multiple Modality and Stages of Usage}

The interactive presentation of online gambling behaviour data is subject to multiple usage styles and modalities at different stages, e.g. self-monitoring and self-regulation as part of an after-care process; or an illustration of addictive patterns as part of a gambler's enrolment with a gambling system. This provides an opportunity for individuals to gain more awareness and control over their gambling experience at different stages of the behaviour change.

\section{Principle 12: Freedom of Sharing}

Online data are under the control of the gambling operator and, subject to the principles of the data protection regulation (such as GDPR in EU [63]) being adhered to, the operator has the right to exploit data commercially. At the same time, gamblers shall not be prevented from obtaining and sharing these data with the parties they consider legitimate.

\section{Proposed Architecture for Persuasive Responsible Online Gambling}

The conceptual architecture of our vision is presented in Figure 3. In order to meet all of the requirements, debates and issues that were summarised in the previous sections, we propose a hybrid approach that composes two complete subsystems: (1) an intelligent end-user agent and (2) a research platform for responsible online gambling. The research platform is responsible for supporting researches about persuasive technologies that could be applied to individuals with addictive behaviour. After a trial and evaluation period, the findings (from (2)) could then feed the intelligent end-user agent. The intelligent end-user agent can run autonomously to a large extent and is responsible for guiding the gamblers to self-regulate their problematic behaviour. Its main advantage is that it provides high privacy guarantees to the individuals because the storage of data and the processing happens on the user-side without any leakages to third-party systems.

In more detail, the architecture of the research platform consists of the following:

- Central repository: The central repository is responsible only for the temporary storage of gamblers' data for research purposes. This data can be divided into two main categories, (a) online gambling behavioural data and (b) multimodal data. The first category is about data that are recorded by the gambling operators, such as betting history, spent time, amount of money and online status (more details about the availability of this data are discussed in Subsection 7.2). The second category is about data that are captured by the gambler's personal devices, such as geolocation, accelerometer data, eye tracking data and heart rate. The stored data in this repository would be derived from multiple gamblers and operators to cover a holistic view of gamblers' online activity. All of this knowledge is important for research and development purposes, but concurrently it is also risky for privacy, commercialisation and unfair competition reasons. Therefore, this data should be temporarily stored for small period, under operators and gamblers' agreements, and for small-scale application. 


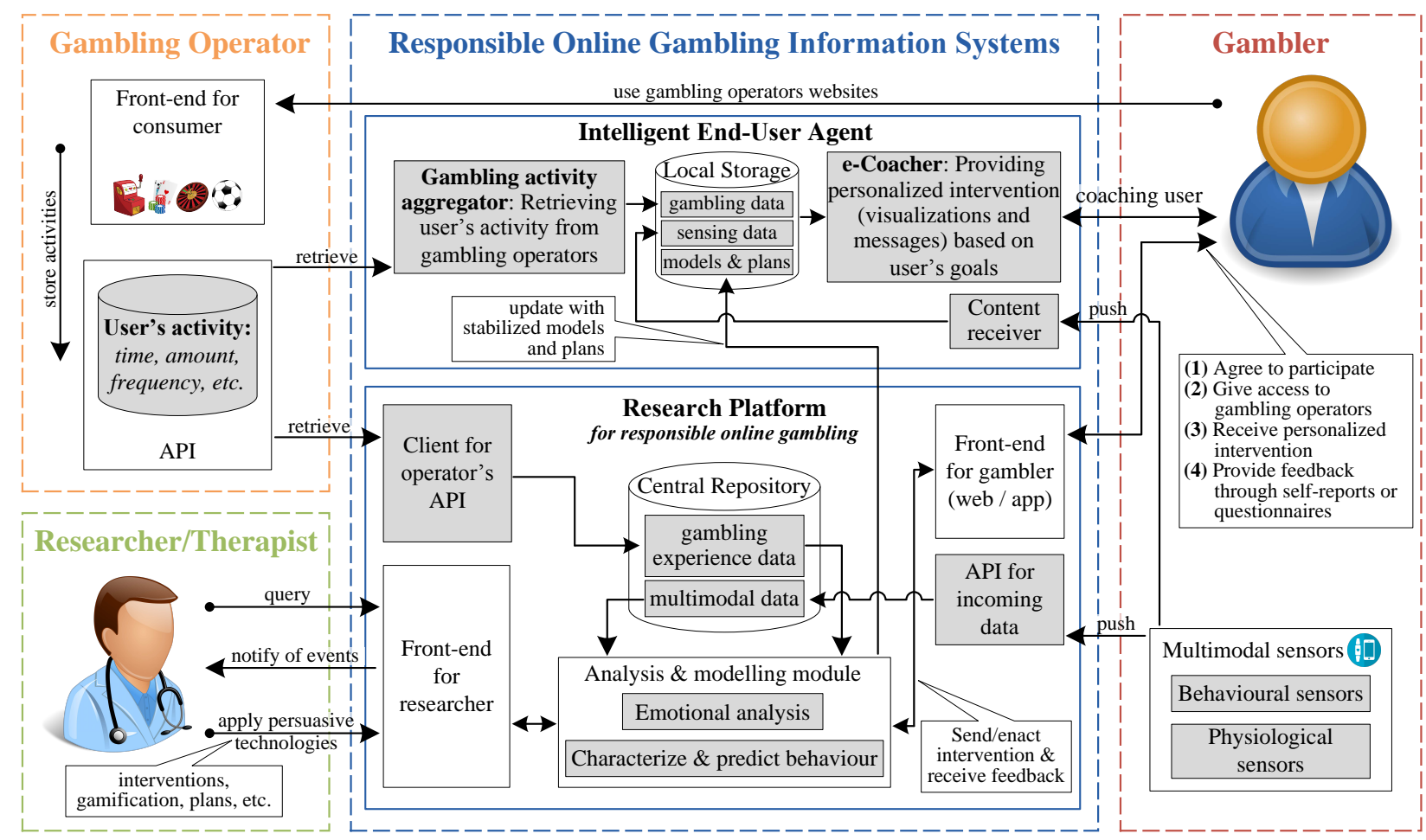

Figure 3. The conceptual architecture for persuasive responsible gambling information systems

- Client for operator's API: A background service that manages gamblers' credentials (e.g. OAuth 2.0 authorisation framework) and retrieves gamblers' activity from online gambling operators' APIs. The frequency of requests to these APIs is important in order to support real-time intervention to gamblers. Ideally, a push API would be the best option for the proposed architecture, but, based on our knowledge, such APIs are not provided by the gambling operators for public use.

- API for incoming data: This API is provided by the research platform in order to collect multimodal sensors' data from the personal devices of gamblers. Thus, an app/service that is running in gambler's mobile phone collects data from the available sensors (i.e., accelerometer, GPS, camera, microphone, etc.) and sends it to the research platform. Apart from this data, we could also aggregate data from wearable sensors (i.e. heart rate, blood pressure, etc.) that store their data to manufactures' cloud (e.g. Fitbit).

- Front-end for researcher/therapist: The researchers' front-end constitutes a web-based interface where a researcher or therapist is able to monitor the gambler's activity, receive notifications, when an irregularity detected on gambler's behaviour, and administer what interventions, gamifications and plans can be applied to each gambler or group of gamblers. In this interface, each gambler has a unique identifier that is not linked, for privacy reasons, with the real identity of gambler and it is only used to trace gamblers' activity.

- Front-end for gambler: The gamblers' front-end could be a web interface and/or a mobile application where the gamblers could watch their betting activity (i.e. time, money, bets, etc.), set the goals that they want to achieve for a healthier gambling activity and receive interventions through suitable visualisations and messages. All of these functionalities are needed to self-regulate their behaviour. In addition to the feedback that can be obtained by the online activity of the gambler, the research platform also requires feedback directly from them, in the form of self-reports or even questionnaires and scales. This feedback will be used by the researchers to conform the applied persuasive technologies and predictive models.

- Analysis and modelling module: This module is the core of the research platform and is responsible for performing all of the necessary analysis and modelling of gamblers' 
multimodal data based on the researchers' instructions. This module mainly consists of two sub-components:

- Emotional analysis: This sub-component is responsible for processing and analysing the signals captured by the corresponding sensors and for generating and utilising the emotion models. Additionally, it has to deal with time synchronization of all modalities and alignment of the measurements [41], [43], [71]. The challenge in developing emotion models relies on accounting for the different reaction times of different modalities, the different duration of different emotions and periods of loss of control, as well as the data availability for building the corresponding models.

- Characterize \& predict behaviour: In this sub-component, the extracted emotional data, from the previous sub-component, combined with gambling experience data will be utilised to characterize and predict the gamblers' behaviour. The results of this process would be provided as personalised interventions (i.e. visualisations and notifications) to the gamblers with the goal to inform and empower them, and eventually help them to change their behaviour.

Finally, after a trial and evaluation period the generated models and algorithms will be applied to the intelligent end-user agent that operates autonomously and targets the general population of gamblers.

Accordingly, the intelligent end-user agent, based on the current technological developments, could be provided as a mobile application or even a client-side web application (an example of both approaches is presented in [72]). The usage of smartphones as a platform to serve the intelligent agent through a mobile application could be the best option, because it is a well-known and powerful computing platform, is continuously together with the gambler, and embeds multiple sensors. The description of components that compose the intelligent end-user agent is as follows:

- Local storage: The local storage is a small database inside the intelligent agent that only stores the personal data of gambler and knowledge about the last up-to-date predictive models. These models are applied, only when previously validated and confirmed, by the research platform.

- Gambling activity aggregator: A service that runs in the background and aggregates gamblers' activity from online gambling operators' APIs. Furthermore, it could be run occasionally when a network is available and with a fixed frequency of requests.

- Content receiver: The content receiver is an interface that provides access to sensor data (e.g. geolocation and accelerometer) from the gambler's personal devices and/or retrieves data, through content providers or an API, from other third-party applications that manage wearable sensors (e.g. Fitbit).

- e-Coacher: The e-Coacher is the core component of the intelligent end-user agent and is responsible for applying the available predictive models and algorithms on the gambler's data. The results of this processing are then used to provide personalised interventions through visualisations and notifications. This personalisation happens using the gambler's profile data, betting activity and the goals that gamblers have set for themselves. Thus, the gamblers have a continuous interaction with their e-Coachers to help them achieve a healthier gambling behaviour.

\subsection{Authentication Process for Gamblers' Participation}

The major challenges facing the feasibility of the proposed architecture are privacy, informed consent and authentication issues. This is mostly about the sharing of online gambling behavioural data with third-parties (e.g. researchers or therapists, who recruit gamblers to participate in a study or trial) even when they are authorised by the gamblers and reviewed by the gambling operator for their intent and declared data usage. In order to assure the participation of gamblers in the research platform, we suggest a privacy-by-design procedure that assures the gambling operators about the 
gamblers' intent to participate in the research platform and facilitate the handling of anonymity and the effect of data leakage particularly when storing the retrieved gambling behavioural data on the side of a third-party provider or cloud storage. The overall procedure is shown in Figure 4. A detailed description of this procedure is presented as follows:

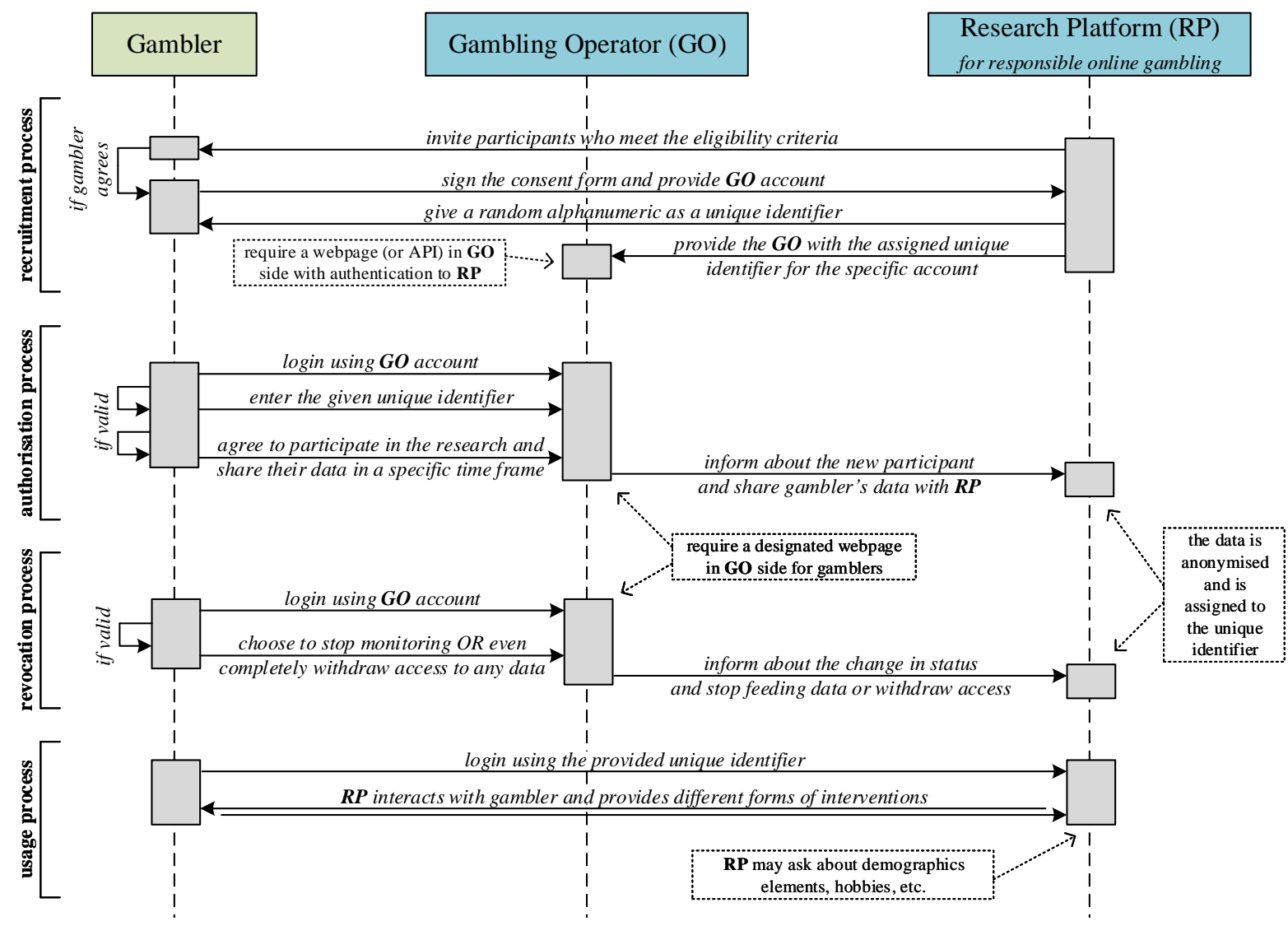

Figure 4. The diagram of a consensus procedure for the gamblers' participation

- The moderator (researcher or therapist) using the research platform will start by inviting participants who meet the eligibility criteria to participate.

- The gamblers who want to participate in the platform and a specific study, shall first sign the consent form and give their account to a gambling operator (or accounts to multiple operators) on the platform. Then, they will receive a random alphanumeric as a unique identifier for the particular study or therapy.

- The moderator, researcher or therapist, should then provide the gambling operator (through a dedicated web page or programmatically via an API) with the unique identifier for the particular account.

- After that, the gamblers shall be instructed to login with their account into the gambling operator and visit a designated web page in the gambling site itself where they have to insert the provided unique identifier. If it matches with their account, they will be requested to agree (or not) to participate in the platform and share their data (for a specific time frame, including the past) with the moderator. At the end of this procedure, the gambling operator will inform the research platform about the new participant.

- Anytime, the gamblers can visit the same page and choose to stop the monitoring process, preventing the moderator from seeing their gambling behaviour. Additionally, they may choose to withdraw access to the data already collected and made available to the moderator. In both cases, the platform will be informed by the gambling operator for this change in the status. 
- The gamblers can then use the website and app provided by the research platform by entering their unique identifier. None of the messages sent to them will contain personally identifiable information (PII).

This protocol seems to be acceptable by the various parties involved in the procedure, i.e. the moderators, the gamblers and the gambling operators. The reliance on the strength of the authentication process at the operators' side is a key part of it. The storage of anonymised data at the platform side, where the mapping between the unique identifiers and the real identities of gamblers is stored separately, is also an additional assurance against data leakage and its effect.

\subsection{Availability of Gambling Operators Data}

The online gambling operators currently provide infrastructures where gamblers can access their personal data. These infrastructures consist mainly of publicly available APIs and data exporting mechanisms through the operators' websites (e.g. download as *.csv files). According to the European data protection regulations (see more in Section 5), each individual has the right to know what data and under what circumstances an operator keeps about them and also has the right to access or even delete this data. Unfortunately, the existing infrastructures provide only some of the gamblers' personal data that describe their online gambling activity. This data mostly includes the following:

- Betting history: A list of the gambler's bets that comprises data such as: date and time, a general description of the bet, status of the bet (won or lost), stake and pot return.

- Account statements: A list of transactions in the gambler's virtual wallet that comprises data such as: date and time, description of the transaction (e.g. casino, sportsbook, etc.), debit or credit amount and balance.

These types of data can be used in the proposed architecture, in addition to informing the gamblers about their betting activity and spending, to acquire knowledge about their level in the gambling addiction, whether there are any increasing or decreasing trends and time consumed (without good accuracy) in gambling. However, this data is insufficient to enable accurate, smart and meaningful interventions to the gamblers. Thus, it is vital for the gambling operators to enrich their infrastructures (and especially APIs) by providing more details about gamblers' online activity. Note that this is data that is already recorded for internal reasons (e.g. security and marketing). For instance, this supplemental data could be:

- Additional details about betting history: This comprises the activities with the date and time, type of game and type of event where the gamblers place their bets (i.e. football, UEFA Europa league, top popular, etc.). Furthermore, it would be good to know the medium used to access the operator (mobile app or website), the gambler's location, whether a bet is cashed out, the time of the event and its location. This analytic betting history could then be used in order to provide more appropriate messages based on the current gambler's context and to predict, for instance, how the gambler bets in comparison with their location. Thus, this inside information could make the gambler to change behaviour more easily.

- Real-time navigation behaviour in operators' platforms: This comprises information about the state of the gambler (i.e. logged in or logged out), the visited areas in the operators' platforms (e.g. pages such as casino, account and betting history, bingo, sportsbook, etc.), the navigation spending time and the clicked links which redirect to third-party web pages (e.g. responsible gambling sites, etc.). In addition to the previous required data from gambling operators, the real-time navigation behaviour of the gambler could help in sending messages or notifications in appropriate times, for instance, just before placing a new bet. 


\section{Discussion and Research Challenges}

Gambling businesses develop complex strategies to utilise and analyse customer' data. Maximising user experience, trading, marketing and overall commercial strategies have been the predominant objectives of individual operators. At this moment, considering the ever increasing regulatory burden as well as raising awareness of social responsibility, it is more demanding than ever to engage in collaborative projects to research and use online data for gambler protection purposes [70], [73]. Responsible gambling is recognised as an area of need, and given the variety of available data, it offers a considerable opportunity to link the identification of problem gambling with appropriate communications, timed and constructed to fit individuals and their particular circumstances. There is an acknowledgment of the challenges surrounding the exchange of personal data and obtaining relevant authorisations on a legal level, as well as the antagonistic nature of the industry, however in consideration of benefits to be acquired by engaging in this area, not only to facilitate a safer gambling experience for the individuals, but also to support responsible gambling strategies across the industry. Maintaining a healthy balance between commercial and responsible gambling requirements can be achieved by relevant investments in research and technological solutions that support both the facilitation of decision-making with more information as well as the progressive interaction and intervention programs.

While digital technologies can be utilised to facilitate and enhance behaviour change strategies there are also risks that need to be taken into account. One of these risks is associated with unsustainable change. Goal setting theory argues that, learning goals relate to improving competencies by targeting skills and acquiring knowledge, whereas performance goals focus on rewards to provide a sense of accomplishment [74]. Learning goals are more effective in achieving a permanent change of attitude, as they can motivate the pursuit of goal when faced with failure [75]. In the short term, users are more likely to be actuated by performance goals, which may have the undesirable effect of decreasing motivation. For instance, if an individual is consistently at the bottom of a leader board, they may develop a sense of low self-efficacy, in which they cease to believe that they are able to achieve a behaviour change. According to social psychological theories, such as learned helplessness [76], this may mean that the individual stops even trying to change and disengages with the process. Likewise, attempts to promote a social norm within a group may be counterproductive if there is a sub-group that defines itself by behaving in an extreme way, as is the case of alcohol use in some populations [77].

Another issue is loss of interest. Whilst online interventions are likely to reach a wide population, attrition is common and tends to occur quickly [78]. The challenge is how to enable a permanent behaviour change. Failure to engage is another common threat. This may be due to negative feelings faced by individuals when they do not receive anticipated support from peers. Careful expectation management is essential in order to manage such risks. Comparison techniques are part of multiple digital interventions. Comparisons are performed with previous behaviour, peers or in relation to the goals set. Comparing behaviour to past behaviour or behaviour of others is intended to enhance self-esteem and self-efficacy. However, such a strategy may negatively affect those constructs. For instance, upward social comparisons (with those with less problematic use) may lead to an individual feeling depressed and unmotivated [7].

Although an online intervention that encourages people to gamble responsibly would usually be considered positive, it is possible that such an intervention may become the basis for alternative addictive behaviours [7]. For instance, email or text messages from the software may cause further gambling incidents. It is also possible that having a leader board may lead individuals checking websites more, as well as becoming obsessed with their position. While this would not congest loss of money in the same way as online gambling, it may be equally problematic in terms of time loss.

Whilst individuals are allowed to set their own goals, this can enhance self-efficacy, but SMART [24] goals are difficult to determine. In the development of a web-based weight loss intervention, users struggled with setting SMART goals [79]. Users may be affected by previous experiences; 
and previous experience of setting challenging goals that have led to failure, may lead them to setting easy goals. Further, when setting goals in order to achieve a specific position on a leader board, individuals may set easy goals, especially if they compare themselves to those with more problematic use (downward comparison). Any platform developed therefore needs to provide examples of SMART goals, ideally tailored to the individual's situation.

\section{Conclusions}

The aim of this article is to shed light on an emerging paradigm of information systems that is meant to support behavioural awareness and behavioural change via persuasive and motivational software-based techniques. Particularly, we study behaviours which can be tracked, to some extent, through automated means, e.g. online presence and sport activities, etc. We take as an exemplary area the case of online gambling. We argue that responsible online gambling can be enriched by persuasive techniques that make use of the gamblers online behavioural, emotional and profile data. Such technology can increase the potential of behaviour change techniques, but may also have unpredictable consequences. To avoid these consequences, developing impartial ways of measuring emotions and creating emotions to the persuasive design has the potential to deploy better, targeted and personalised interventions for gamblers with a problematic behaviour. From the perspective of gambling operators, we would require them to exhibit greater social responsibility, in particular, with a collaborative approach to data sharing and management.

The article is built with our practical experience and joint effort with main gambling addiction recovery charities and responsible gambling experts in the UK. We make the point that data, which is already available to the operators and used for their own commercial purposes, should be equally disposable for responsible gambling reasons. Data protection regulations reveal that this is the right time for online gambling operators to start sharing their data. To this end, we present the required principles that should be followed by the gambling industry to enable responsible online gambling. Additionally, we propose a conceptual architecture that shows our vision in this domain and demonstrates how such approaches could be developed. However, the main objective of this article is to provide a starting point for further discussion on these issues, which would ideally involve contributions from the perspective of computing, psychology and corporate social responsibility. This would encourage, what we think is much needed, policy change in this area, or, as a minimum, engender a shared corporate view which drives to the provision of the kind of data that could be used to enable systems, such as those described in the concept, to mitigate against problematic gambling.

Despite the focus on online gambling in this article, much of the presented foundations and discussions apply equally to persuasive technologies in general, for combatting problematic online behaviour. For instance, game addiction, persistent use of social media, compulsive online shopping are other domains where we can demand that online behavioural data are provided available to the gamblers and their surrogate agent, counsellor or software. Although such data sharing is often encouraged for transparency and informed usage reasons, challenges remain in relation to its potential of affecting gamblers experience and also causing negative side-effects. This requires more theory-informed utilisation of data to design the interaction, persuasive and motivational mechanisms over the life cycle of change.

\section{Acknowledgments}

This work was partly supported by the EROGamb Project (Empowering Responsible Online Gambling with Predictive, Real-time, Persuasive and Interactive Intervention). This project is funded jointly by GambleAware and Bournemouth University. We also thank The Gambling Therapy for sharing its practical experience in the field of problem gambling and providing feedback on this work. 


\section{References}

[1] American Psychiatric Association, Diagnostic and statistical manual of mental disorders (DSM-5), 5th ed. Washington, DC: American Psychiatric Association Press, 2013.

[2] Gambling Commission, "Industry statistics April 2014 to March 2017," 2017, accessed 10 Oct. 2018. [Online]. Available: http://www.gamblingcommission.gov.uk/PDF/survey-data/ Gambling-industry-statistics.pdf

[3] L. Davidson, M. Chinman, B. Kloos, R. Weingarten, D. Stayner, and J. K. Tebes, "Peer support among individuals with severe mental illness: A review of the evidence," Clinical Psychology: Science and Practice, vol. 6, no. 2, pp. 165-187, 2006. [Online]. Available: https://doi.org/10.1093/clipsy.6.2.165

[4] R. H. Moos and B. S. Moos, "Help-seeking careers: Connections between participation in professional treatment and alcoholics anonymous," Journal of Substance Abuse Treatment, vol. 26, no. 3, pp. 167-173, 2004. [Online]. Available: https://doi.org/10.1016/S0740-5472(03)00190-9

[5] R. Ali, N. Jiang, K. Phalp, S. Muir, and J. McAlaney, "The emerging requirement for digital addiction labels," in Requirements Engineering: Foundation for Software Quality, S. A. Fricker and K. Schneider, Eds. Cham: Springer International Publishing, pp. 198-213, 2015. [Online]. Available: https://doi.org/10.1007/978-3-319-16101-3_13

[6] A. Alrobai, J. McAlaney, K. Phalp, and R. Ali, "Online peer groups as a persuasive tool to combat digital addiction," in Persuasive Technology, A. Meschtscherjakov, B. De Ruyter, V. Fuchsberger, M. Murer, and M. Tscheligi, Eds. Cham: Springer International Publishing, pp. 288-300, 2016. [Online]. Available: https://doi.org/10.1007/978-3-319-31510-2_25

[7] A. Alrobai, J. McAlaney, K. Phalp, and R. Ali, "Exploring the risk factors of interactive e-health interventions for digital addiction," International Journal of Sociotechnology and Knowledge Development, vol. 8, no. 2, pp. 1-15, 2016. [Online]. Available: https://doi.org/10.4018/IJSKD. 2016040101

[8] I. Ajzen, "The theory of planned behavior," Organizational behavior and human decision processes, vol. 50, no. 2, pp. 179-211, 1991. [Online]. Available: https://doi.org/10.1016/0749-5978(91)90020-T

[9] S. Leigh and S. Flatt, “App-based psychological interventions: friend or foe?” Evidence-Based Mental Health, 2015. [Online]. Available: https://doi.org/10.1136/eb-2015-102203

[10] T. J. Dishion, J. McCord, and F. Poulin, "When interventions harm: Peer groups and problem behavior." American psychologist, vol. 54, no. 9, pp. 755-764, 1999. [Online]. Available: https://doi.org/10.1037/0003-066X.54.9.755

[11] S. Karau and D. Williams, "Social loafing: A meta-analytic review and theoretical integration," Journal of Personality and Social Psychology, vol. 65, pp. 681-706, 10 1993. [Online]. Available: https://doi.org/10.1037//0022-3514.65.4.681

[12] V. L. Allen, "Situational factors in conformity," Advances in experimental social psychology, vol. 2, pp. 133-175, 1965. [Online]. Available: https://doi.org/10.1016/S0065-2601(08)60105-7

[13] J. Hamari, J. Koivisto, and T. Pakkanen, "Do persuasive technologies persuade? - A review of empirical studies," in Persuasive Technology. Cham: Springer International Publishing, pp. 118-136, 2014. [Online]. Available: https://doi.org/10.1007/978-3-319-07127-5_11

[14] M. D. Griffiths, "Internet gambling, player protection, and social responsibility," in Routledge international handbook of Internet gambling, R. J. Williams, R. T. Wood, and J. Parke, Eds. London and New York: Routledge, ch. 13, pp. 227-249, 2012.

[15] R. W. Rogers, "Cognitive and psychological processes in fear appeals and attitude change: A revised theory of protection motivation," in Social psychophysiology: A sourcebook, J. T. Cacioppo and R. E. Petty, Eds. New York, USA: Guilford Press, Sept., pp. 153-176, 1983. 
[16] L. Ross, "The intuitive psychologist and his shortcomings: Distortions in the attribution process," ser. Advances in Experimental Social Psychology, L. Berkowitz, Ed. Academic Press, vol. 10, pp. 173 220, 1977. [Online]. Available: https://doi.org/10.1016/s0065-2601(08)60357-3

[17] T. L. Webb and P. Sheeran, "Does changing behavioral intentions engender behavior change? A meta-analysis of the experimental evidence," Psychological Bulletin, vol. 132, no. 2, pp. 249-268, 2006. [Online]. Available: https://doi.org/10.1037/0033-2909.132.2.249

[18] J. O. Prochaska, , and C. DiClemente, "Stage of change in the modification of problem behaviors," Progress in behavior modification, vol. 28, pp. 184-218, 1992.

[19] I. J. Reagan, J. P. Bliss, R. V. Houten, and B. W. Hilton, "The effects of external motivation and real-time automated feedback on speeding behavior in a naturalistic setting," Human Factors, vol. 55, no. 1, pp. 218-230, 2013. [Online]. Available: https://doi.org/10.1177/0018720812447812

[20] P. M. Gollwitzer, "Action phases and mindsets," in Handbook of motivation and cognition: Foundations of social behavior, E. Higgins and R. M. Sorrentino, Eds. New York, USA: Guilford Press, June, vol. 2, pp. 53-92, 1990.

[21] E. L. Deci, R. Koestner, and R. M. Ryan, "A meta-analytic review of experiments examining the effects of extrinsic rewards on intrinsic motivation," Psychological Bulletin, vol. 125, no. 6, pp. 627-668, 1999. [Online]. Available: https://doi.org/10.1037/0033-2909.125.6.627

[22] R. M. Ryan and E. L. Deci, "Self-determination theory and the facilitation of intrinsic motivation, social development, and well-being," American psychologist, vol. 55, no. 1, p. 68, 2000. [Online]. Available: https://doi.org/10.1037/0003-066X.55.1.68

[23] G. H. Seijts, G. P. Latham, K. Tasa, and B. W. Latham, "Goal setting and goal orientation: An integration of two different yet related literatures," Academy of management journal, vol. 47, no. 2, pp. 227-239, 2004. [Online]. Available: https://doi.org/10.5465/20159574

[24] G. T. Doran, “There's a smart way to write management's goals and objectives," Management review, vol. 70 , no. 11 , pp. 35-36, 1981.

[25] L. K. B. Eldredge, G. S. Parcel, G. Kok, N. H. Gottlieb, and M. E. Fernández, Planning health promotion programs: an intervention mapping approach, 3rd ed. San Francisco, CA: Jossey-Bass, 2011.

[26] P. M. Gollwitzer and P. Sheeran, "Implementation intentions and goal achievement: A meta-analysis of effects and processes," Advances in experimental social psychology, vol. 38, pp. 69-119, 2006. [Online]. Available: https://doi.org/10.1016/S0065-2601(06)38002-1

[27] L. Festinger, “A theory of social comparison processes," Human Relations, vol. 7, no. 2, pp. 117-140, 1954. [Online]. Available: https://doi.org/10.1177/001872675400700202

[28] R. N. Landers, K. N. Bauer, and R. C. Callan, "Gamification of task performance with leaderboards: A goal setting experiment," Computers in Human Behavior, vol. 71, no. Supplement C, pp. 508-515, 2017. [Online]. Available: https://doi.org/10.1016/j.chb.2015.08.008

[29] J. McAlaney, B. Bewick, and C. Hughes, "The international development of the 'social norms' approach to drug education and prevention," Drugs: Education, Prevention and Policy, vol. 18, no. 2, pp. 81-89, 2011. [Online]. Available: https://doi.org/10.3109/09687631003610977

[30] L. Marchica and J. L. Derevensky, "Examining personalized feedback interventions for gambling disorders: A systematic review," Journal of behavioral addictions, vol. 5, no. 1, pp. 1-10, 2016. [Online]. Available: https://doi.org/10.1556/2006.5.2016.006

[31] T. Ricketts and A. Macaskill, "Gambling as emotion management: Developing a grounded theory of problem gambling," Addiction Research \& Theory, vol. 11, no. 6, pp. 383-400, 2003. [Online]. Available: https://doi.org/10.1080/1606635031000062074 
[32] A. Blaszczynski and L. Nower, "A pathways model of problem and pathological gambling," Addiction, vol. 97, no. 5, pp. 487-499, 2002. [Online]. Available: https://doi.org/10.1046/j.1360-0443. 2002.00015.x

[33] M. Di Trani, A. Renzi, C. Vari, G. C. Zavattini, and L. Solano, "Gambling disorder and affect regulation: The role of alexithymia and attachment style," Journal of Gambling Studies, vol. 33, no. 2, pp. 649-659, Jun 2017. [Online]. Available: https://doi.org/10.1007/s10899-016-9637-3

[34] A. Estévez, P. Jáuregui, I. Sánchez-Marcos, H. López-González, and M. D. Griffiths, "Attachment and emotion regulation in substance addictions and behavioral addictions," Journal of behavioral addictions, vol. 6, no. 4, pp. 534-544, Dec 2017. [Online]. Available: https://doi.org/10.1556/2006.6. 2017.086

[35] G. Rogier and P. Velotti, "Conceptualizing gambling disorder with the process model of emotion regulation," Journal of behavioral addictions, vol. 7, no. 2, p. 239-251, June 2018. [Online]. Available: https://doi.org/10.1556/2006.7.2018.52

[36] C. Bonnaire, C. Bungener, and I. Varescon, "Alexithymia and gambling: A risk factor for all gamblers?” Journal of Gambling Studies, vol. 29, no. 1, pp. 83-96, Mar 2013. [Online]. Available: https://doi.org/10.1007/s10899-012-9297-x

[37] L. Lole, C. J. Gonsalvez, A. Blaszczynski, and A. R. Clarke, "Electrodermal activity reliably captures physiological differences between wins and losses during gambling on electronic machines," Psychophysiology, vol. 49, no. 2, pp. 154-163, 2012. [Online]. Available: https: //doi.org/10.1111/j.1469-8986.2011.01290.x

[38] B. Studer and L. Clark, "Place your bets: psychophysiological correlates of decision-making under risk," Cognitive, Affective, \& Behavioral Neuroscience, vol. 11, no. 2, pp. 144-158, Jun 2011. [Online]. Available: https://doi.org/10.3758/s13415-011-0025-2

[39] S. Sharman and L. Clark, "Mixed emotions to near-miss outcomes: A psychophysiological study with facial electromyography," Journal of Gambling Studies, vol. 32, no. 3, pp. 823-834, Sep 2016. [Online]. Available: https://doi.org/10.1007/s10899-015-9578-2

[40] B. D. Nelson, E. M. Kessel, F. Jackson, and G. Hajcak, "The impact of an unpredictable context and intolerance of uncertainty on the electrocortical response to monetary gains and losses," Cognitive, Affective, \& Behavioral Neuroscience, vol. 16, no. 1, pp. 153-163, Feb 2016. [Online]. Available: https://doi.org/10.3758/s13415-015-0382-3

[41] T. Kostoulas, G. Chanel, M. Muszynski, P. Lombardo, and T. Pun, "Films, affective computing and aesthetic experience: Identifying emotional and aesthetic highlights from multimodal signals in a social setting," Frontiers in ICT, vol. 4, p. 11, 2017. [Online]. Available: https://doi.org/10.3389/fict. 2017.00011

[42] T. Kostoulas, G. Chanel, M. Muszynski, P. Lombardo, and T. Pun, "Identifying aesthetic highlights in movies from clustering of physiological and behavioral signals," in Quality of Multimedia Experience (QoMEX), 2015 Seventh International Workshop on. IEEE, pp. 1-6, 2015. [Online]. Available: https://doi.org/10.1109/QoMEX.2015.7148098

[43] T. Kostoulas, I. Mporas, O. Kocsis, T. Ganchev, N. Katsaounos, J. J. Santamaria, S. Jimenez-Murcia, F. Fernandez-Aranda, and N. Fakotakis, "Affective speech interface in serious games for supporting therapy of mental disorders," Expert Systems with Applications, vol. 39, no. 12, pp. 11 072-11 079, 2012. [Online]. Available: https://doi.org/10.1016/j.eswa.2012.03.067

[44] F. Eyben, F. Weninger, F. Gross, and B. Schuller, "Recent developments in opensmile, the munich open-source multimedia feature extractor," in 21st ACM International Conference on Multimedia, ser. MM '13. New York, NY, USA: ACM, pp. 835-838, 2013. [Online]. Available: https://doi.org/10.1145/2502081.2502224 
[45] G. Bradski and A. Kaehler, Learning OpenCV: Computer vision with the OpenCV library. O'Reilly Media, Inc., 2008.

[46] T. Baltrušaitis, P. Robinson, and L.-P. Morency, "OpenFace: An open source facial behavior analysis toolkit," in IEEE Winter Conference on Applications of Computer Vision (WACV), March, pp. 1-10, 2016. [Online]. Available: https://doi.org/10.1109/WACV.2016.7477553

[47] T. Kostoulas, G. Chanel, M. Muszynski, P. Lombardo, and T. Pun, "Dynamic time warping of multimodal signals for detecting highlights in movies," in Proceedings of the 1st Workshop on Modeling INTERPERsonal SynchrONy And infLuence, ser. INTERPERSONAL '15. New York, NY, USA: ACM, pp. 35-40, 2015. [Online]. Available: https://doi.org/10.1145/2823513.2823515

[48] L. Tian, M. Muszynski, C. Lai, J. Moore, T. Kostoulas, P. Lombardo, T. Pun, and G. Chanel, "Recognizing induced emotions of movie audiences: Are induced and perceived emotions the same?" in 7th International Conference on Affective Computing and Intelligent Interaction (ACII). IEEE, pp. 1-8, 2017. [Online]. Available: https://doi.org/10.1109/ACII.2017.8273575

[49] E. Kroupi, J.-M. Vesin, and T. Ebrahimi, "Phase-amplitude coupling between EEG and EDA while experiencing multimedia content," in Humaine Association Conference on Affective Computing and Intelligent Interaction (ACII). IEEE, Sept, pp. 865-870, 2013. [Online]. Available: https: //doi.org/10.1109/ACII.2013.162

[50] T. Joachims, "Making large-scale svm learning practical," Dortmund, Technical Report, SFB 475: Komplexitätsreduktion in Multivariaten Datenstrukturen, Universität Dortmund 1998, 28, 1998. [Online]. Available: http://hdl.handle.net/10419/77178

[51] L. Rabiner and B. Juang, "An introduction to hidden markov models," IEEE ASSP Magazine, vol. 3, no. 1, pp. 4-16, Jan 1986. [Online]. Available: https://doi.org/10.1109/MASSP.1986.1165342

[52] H. P. Martinez, Y. Bengio, and G. N. Yannakakis, "Learning deep physiological models of affect," IEEE Computational Intelligence Magazine, vol. 8, no. 2, pp. 20-33, May 2013. [Online]. Available: https://doi.org/10.1109/MCI.2013.2247823

[53] A. Harris, A. Parke, and M. D. Griffiths, "The case for using personally relevant and emotionally stimulating gambling messages as a gambling harm-minimisation strategy," International Journal of Mental Health and Addiction, vol. 16, no. 2, pp. 266-275, 2016. [Online]. Available: https://doi.org/10.1007/s11469-016-9698-7

[54] M. M. Auer and M. D. Griffiths, "The use of personalized behavioral feedback for online gamblers: an empirical study," Frontiers in psychology, vol. 6, p. 1406, 2015. [Online]. Available: https://doi.org/10.3389/fpsyg.2015.01406

[55] J. Torous, "Digital psychiatry," IEEE Spectrum, vol. 54, no. 7, pp. 45-50, 2017. [Online]. Available: https://doi.org/10.1109/MSPEC.2017.7951722

[56] D. Miers, "Social responsibility and harm minimization in commercial gambling in great britain," Gaming Law Review and Economics, vol. 20, no. 2, pp. 164-176, 2016. [Online]. Available: https://doi.org/10.1089/glre.2016.2024

[57] Gambling Commission, "Gambling participation in 2016: behavior, awareness and attitudes - Annual report," 2017, accessed 10 Oct. 2018. [Online]. Available: http://www.gamblingcommission.gov.uk/ PDF/survey-data/Gambling-participation-in-2016-behaviour-awareness-and-attitudes.pdf

[58] E. Korkea-Aho and P. Leino, "Who owns the information held by EU agencies? Weed killers, commercially sensitive information and transparent and participatory governance," Common Market Law Review, vol. 54, no. 4, pp. 1059-1091, 2017.

[59] J. D. Pratten and S. Walton, "Policy and reality: Corporate social responsibility in the UK gambling industry," in Corporate Responsibility Research Conference, 2008. 
[60] A. Blaszczynski, P. Collins, D. Fong, R. Ladouceur, L. Nower, H. J. Shaffer, H. Tavares, and J.-L. Venisse, "Responsible gambling: General principles and minimal requirements," Journal of Gambling Studies, vol. 27, no. 4, pp. 565-573, Dec 2011. [Online]. Available: https: //doi.org/10.1007/s10899-010-9214-0

[61] T. C. Lin, "Executive trade secrets," Notre Dame L. Rev., vol. 87, p. 911, 2011.

[62] N. Hing, L. Cherney, A. Blaszczynski, S. M. Gainsbury, and D. I. Lubman, "Do advertising and promotions for online gambling increase gambling consumption? An exploratory study," International Gambling Studies, vol. 14, no. 3, pp. 394-409, 2014. [Online]. Available: https: //doi.org/10.1080/14459795.2014.903989

[63] European Parliament and Council, "Regulation (EU) 2016/679 of 27 April 2016 on the protection of natural persons with regard to the processing of personal data and on the free movement of such data, and repealing Directive 95/46/EC (General Data Protection Regulation)," Official Journal of the European Union, p. 88, 2016.

[64] J. Metcalf, E. F. Keller, and D. Boyd, "Perspectives on big data, ethics and society," The Council for Big Data, Ethics and Society, 2016.

[65] T. Y. Tang and P. Winoto, "I should not recommend it to you even if you will like it: the ethics of recommender systems," New Review of Hypermedia and Multimedia, vol. 22, no. 1-2, pp. 111-138, 2016. [Online]. Available: https://doi.org/10.1080/13614568.2015.1052099

[66] D. Paraschakis, "Towards an ethical recommendation framework," in 11th International Conference on Research Challenges in Information Science (RCIS). IEEE, pp. 211-220, 2017. [Online]. Available: https://doi.org/10.1109/RCIS.2017.7956539

[67] R. Cassidy, A. Pisac, and C. Loussouarn, Qualitative research in gambling: Exploring the production and consumption of risk. Routledge, 2013. [Online]. Available: https://doi.org/10.26530/OAPEN_ 610650

[68] Information Commissioner's Office (ICO), "Findings, recommendations and actions from ico investigation into data analytics in political campaigns," July 2018, accessed 12 October 2018. [Online]. Available: https://ico.org.uk/about_the_ico/news_and_events/news_and_blogs/2018/07/findings_ recommendations_and_actions_from_ico_investigation_into_data_analytics_in_political_campaigns/

[69] UK Public General Acts, "Gambling Act 2005,” 2005, accessed 12 Oct. 2018. [Online]. Available: http://www.legislation.gov.uk/ukpga/2005/19/pdfs/ukpga_20050019_en.pdf

[70] Responsible Gambling Strategy Board, "The national responsible gambling strategy 2016-17 to 2018-19," 2016, accessed 12 Oct. 2018. [Online]. Available: https://about.gambleaware.org/media/ 1230/rgsb_strategy_2016-2019.pdf

[71] M. Soleymani, S. Asghari-Esfeden, M. Pantic, and Y. Fu, "Continuous emotion detection using EEG signals and facial expressions," in IEEE International Conference on Multimedia and Expo (ICME), pp. 1-6, 2014. [Online]. Available: https://doi.org/10.1109/ICME.2014.6890301

[72] P. S. Efraimidis, G. Drosatos, A. Arampatzis, G. Stamatelatos, and I. N. Athanasiadis, "A privacy-by-design contextual suggestion system for tourism," Journal of Sensor and Actuator Networks, vol. 5, no. 2, 2016. [Online]. Available: https://doi.org/10.3390/jsan5020010

[73] S. Harrison, "Fairer and safer gambling (keynote speech)," in Raising Standards Conference. Gambling Commission, Nov, pp. 1-7, 2017. [Online]. Available: http://www.gamblingcommission. gov.uk/PDF/speeches/Raising-standards-keynote-speech-Sarah-Harrison-2017.pdf

[74] E. A. Locke and G. P. Latham, "New directions in goal-setting theory," Current Directions in Psychological Science, vol. 15, no. 5, pp. 265-268, 2006. [Online]. Available: https: //doi.org/10.1111/j.1467-8721.2006.00449.x 
[75] G. H. Seijts and G. P. Latham, "Learning versus performance goals: When should each be used?" The Academy of Management Executive, vol. 19, no. 1, pp. 124-131, 2005. [Online]. Available: https://doi.org/10.5465/ame.2005.15841964

[76] M. E. Seligman, Helplessness: On depression, development, and death. New York: WH Freeman/Times Books/Henry Holt \& Co, 1975.

[77] M. E. Larimer, D. L. Irvine, J. R. Kilmer, and G. A. Marlatt, "College drinking and the greek system: Examining the role of perceived norms for high-risk behavior," Journal of College Student Development, vol. 38, no. 6, p. 587, 1997.

[78] G. Eysenbach, "The law of attrition," Journal of Medical Internet Research, vol. 7, no. 1, p. e11, 2005. [Online]. Available: https://doi.org/10.2196/jmir.7.1.e11

[79] L. Yardley, S. Williams, K. Bradbury, G. Garip, S. Renouf, L. Ware, H. Dorling, E. Smith, and P. Little, "Integrating user perspectives into the development of a web-based weight management intervention," Clinical Obesity, vol. 2, no. 5-6, pp. 132-141, 2012. [Online]. Available: https://doi.org/10.1111/cob.12001 